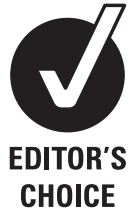

doi: $10.1136 /$ ebm 1068

Technical University of Munich, Munich, Germany

Correspondence to:

Kurt G Naber

Karl-Bickleder-Str. 44c, 94315

Straubing, Germany;

kurt@nabers.de
Randomised controlled trial

\section{Targeted, immediate antibiotics following a positive dipstick test may be the optimal management strategy}

\section{Kurt G Naber}

Commentary on: Little P, Moore MV, Turner S, et al. Effectiveness of five different approaches in management of urinary tract infection: randomised controlled trial. BMJ 2010;340:c199.

This interesting, prospective, and randomised clinical study tested five different approaches in the treatment of women suspected with uncomplicated urinary-tract infection (UTI): immediate, empirical, antibiotic therapy (AT); delayed (by 48 h), empirical AT; empirical AT offered to patients showing a certain clinical symptom score; empirical AT offered to patients with positive dipstick urinalysis; and targeted AT offered according to the results of a midstream urine microbiological culture and symptomatic treatment up to the point when the microbiological results became available.

The study included 309 non-pregnant women aged between 18 and 70 years. All groups were comparable in terms of demographics, clinical characteristics and frequency of symptom severity at the time of study. The study aimed to achieve a similar-level reduction of symptoms without unnecessary antibiotic usage, laboratory investigations and reconsultations. A statistical consideration of sample size was performed for $\alpha=0.05$ and $\beta=0.2$ for multiple groups. Patients with clinical symptoms who did not require immediate AT were included.

The patients had access to antibiotics at their request, despite randomisation. The use of midstream urine culture and dipsticks when not indicated on the advice sheets was also assessed. The study managed to represent the clinical ambulatory situation in a general practice.

According to the analysis, the authors concluded that all five management strategies achieved similar (but not equivalent) symptom reduction; there was no advantage in routinely sending midstream urine samples for (microbiological) testing, and antibiotics targeted with dipsticks with a delayed prescription as backup, or empirical delayed prescription, can help to reduce antibiotic use.

\section{Are these conclusions justified by the data presented?}

The Methods stated that group 1 was offered 'immediate' empirical AT. However, in the Results section it is stated that, on an average, antibiotics were started after 1.19 days in group 1, 1.40 days in group 3 (symptom score) and 1.48 days in group 4 (dipstick urinalysis). Therefore, in none of the three groups was AT commenced immediately and was usually delayed by, at least, 1 day.

The study showed that women who were delayed antibiotic treatment for $48 \mathrm{~h}$ or more were likely to have a $37 \%$ longer duration of symptoms rated as moderately bad $(p<0.001)$. This (statistically significant) difference may be not considered clinically relevant. However, if
AT in groups 1, 3 and 4 had commenced immediately on the day of consultation, and not the next day as the data in the results suggest, it seems likely that this difference should be increased by another day.

Interestingly, in group 1, 97\% used antibiotics, whereas in groups 3 (symptom score) and 4 (dipstick urinalysis) only $90 \%$ and $80 \%$ did so, even though symptom control was similar between groups, and would have been most likely also similar if AT was commenced on the day of consultation. Therefore, preselection of patients with a positive dipstick (if nitrite or leucocytes and a trace of blood is detected in urine) for immediate AT will reduce antibiotic usage without compromise in symptom control. Only patients with a negative dipstick urinalysis should be offered delayed empiric AT, for it is also known that UTI may be present in patients with negative dipstick and these patients may profit more from AT than from placebo.

A previous validation study had shown that dipsticks and clinical scoring algorithms can potentially help improve predictive values of UTI. ${ }^{2}$ The present study confirms the usefulness of this strategy by measuring clinical outcome and antibiotic usage. A shortcoming specific to this study was that a group of patients preselected with dipstick and clinical scoring were not investigated, which may have further reduced the number of patients who were offered immediate AT.

I would like to suggest slightly modified conclusions and recommendations, taking into consideration the cited studies:

1. Early empiric AT as compared with delayed AT of more than $48 \mathrm{~h}$ can help control symptoms faster. Therefore, if AT is indicated, it should be started immediately on the day of consultation. There is no advantage in routinely sending midstream urine samples for microbiological investigations and delay AT while waiting for the results.

2. Immediate empiric AT should be offered only to patients with positive dipstick urinalysis and clinical scoring, in order to minimise unnecessary antibiotic usage.

3. Because patients with negative dipstick urinalysis may profit from AT, delayed AT should be offered to those patients if symptoms persist after $48 \mathrm{~h}$.

This strategy may be the best possible compromise between fast symptom control and reduction of unnecessary antibiotic use.

Competing interests KGNhas been paid as an investigator by MerLion, OM Pharma, Peninsula/Johnson \&t Johnson, Sanofi-Aventis and Zambon. He also has been paid as speaker at scientific meetings/scientific publications by 
Bayer, DaiichiSankyo, Johnson \& Johnson, OM-Pharma, Sanofi-Aventis and Zambon.

\section{References}

1. Richards D, Toop L, Chambers S, et al. Response to antibiotics of women with symptoms of urinary tract infection but negative dipstick urine test results: double blind randomised controlled trial. BMJ 2005;331:143.

2. Little P, Turner S, Rumsby K, et al. Developing clinical rules to predict urinary tract infection in primary care settings: sensitivity and specificity of near patient tests (dipsticks) and clinical scores. Br J Gen Pract 2006;56:606-12. 